\title{
Überlegungen zum Wandel von Arbeit
}

Rainer Trinczek

\begin{abstract}
Über die Zukunft von Arbeit lässt sich trefflich streiten, da in der Tat widersprüchliche Tendenzen empirisch beobachtbar sind: Da ist auf der einen Seite von Prekarisierung und dem Phänomen der „,working poor“die Rede, auf der anderen Seite von Knappheitsphänomenen auf dem Arbeitsmarkt und davon, dass es für eine wachsende Gruppe von (meist hoch qualifizierten) Beschäftigten vor allem um die „Selbstverwirklichung“ bei der Arbeit gehen würde. Im folgenden Beitrag werden einige wesentliche Entwicklungslinien nachgezeichnet, die dieser Pluralisierung der Arbeits- und Beschäftigungsbedingungen zugrunde liegen. ${ }^{1}$
\end{abstract}

\section{Einleitung}

Der Wandel von Arbeit ist ein klassisches Thema (nicht nur) der Arbeits- und Industriesoziologie, und es wäre ein Thema für sich, aus einer gewissen zeitlichen Distanz heraus die Vielzahl an vorliegenden Prognosen aus den letzten Dekaden wieder einmal anzuschauen und rückblickend auf ihren Realitätsgehalt hin zu überprüfen. Dies soll hier allerdings nicht geschehen, sondern es sollen einige Hinweise darauf gesammelt werden, wie sich Arbeit verändert hat und in welcher Richtung sie sich denn wohl in den nächsten Jahren entwickeln dürfte. Dabei versteht es sich von selbst, dass in diesem Beitrag nicht jede Facette des komplexen gesellschaftlichen Phänomens Arbeit angemessen beleuchtet werden kann, sondern eine gezielte Auswahl getroffen werden muss.

Bei der Analyse gegenwärtig beobachtbarer Tendenzen in der Entwicklung von Arbeit ergibt es Sinn, zwischen strukturellen und institutionellen Veränderungen auf gesamtgesellschaftlicher Ebene - gleichsam als Kontext betrieblicher Arbeitspolitiken - und dem Wandel realer Arbeitskonstellationen in der betrieblichen Praxis zu unterscheiden; dass eine solche Unterscheidung eine rein analytische ist, bedarf an dieser Stelle kaum der Erwähnung.

Gleichzeitig ist $\mathrm{zu}$ berücksichtigen, dass spätestens seit Aufkommen des Arbeitspolitik-Ansatzes in den 1980er Jahren bekannt ist, dass der Wandel von Arbeit ein contested terrain (Edwards 1979) ist, sodass Fragen zur Zukunft von Arbeit auch politische Fragen und daher nicht zuletzt stets auch Machtfragen sind.

Der folgende Beitrag wird in drei Schritten vorgehen: Zunächst werden veränderte soziostrukturelle Kontexte von Arbeit (Abschnitt 2) und wichtige Trends in der realen Arbeitspraxis (Abschnitt 3) beschrieben. Was bisherige wie auch erwartbare Veränderungen der Rahmenbedingungen von Arbeit für zukünftige Machtkonstellationen im gesellschaftlichen Feld der Arbeit bedeuten können, wird abschließend im Abschnitt 4 skizziert.

Wie für alle Aussagen über Zukünftiges, so gilt auch für die auf künftige Entwicklungen gerichteten Passagen dieses Beitrags, dass Zukunft prinzipiell kontingent ist; daher ist den entsprechenden Aussagen zwangsläufig ein gewisser spekulativer Charakter zu eigen.

\section{2 \\ Veränderte Kontexte von Arbeit}

Wesentliche Veränderungen im gesamtgesellschaftlichen Kontext von Arbeit betreffen

- die Globalisierung der Ökonomie (2.1), - die Verschiebungen in der Struktur der Ökonomie mit den entsprechenden Folgen für den gesellschaftlichen Qualifikationsbedarf (2.2),

- die Veränderungen der Erwerbstätigenstruktur im Zuge des demografischen Wandels sowie des veränderten Bildungsverhaltens (2.3),

- den Übergang politischer Regulierungen von Arbeit, die typischerweise unter der Überschrift „From Welfare to Workfare“ bearbeitet werden (2.4),

- sowie die Segmentierung der Ökonomie in verschiedene Mitbestimmungs- und Partizipationskonstellationen (2.5).

\subsection{GLOBALISIERUNG DER ÖKONOMIE}

Ohne Zweifel stellt die zunehmende Globalisierung der Ökonomie einen der sogenannten Mega-Trends in der wirtschaftlichen und gesellschaftlichen Entwicklung der letzten Dekaden dar. Die verbesserten Möglichkeiten für Unternehmen, globale Kostendifferenzen für sich zu nutzen und vom internationalen Standortwettbewerb zu profitieren, sind in der Literatur ebenso hinreichend beschrieben wie die daraus erwachsenden Folgen für Arbeit: Entstehung transnationaler Arbeitsräume - teils innerhalb, teils außerhalb von international operierenden Unternehmen; zunehmende Mobilitätsanforderungen an Beschäftigte; wachsende Standortkonkurrenz; zunehmende Interkulturalität in Arbeitsteams etc.

Getrieben ist die Globalisierung nicht zuletzt durch die Liberalisierung des Finanzsektors seit Mitte der 1970er Jahre, dem in der Zwischenzeit eine Leitfunktion innerhalb der Ökonomie zugeschrieben wird (Finanzmarkt-Kapitalismus). Der finanzialisierte Kapitalismus hat - mit Blick auf die Realökonomie - nun nicht nur zahlreiche neue Akteure geschaffen (wie etwa Private-Equity-Fonds etc.), sondern

\footnotetext{
1 Dem anonymen Gutachter bzw. der anonymen Gutachterin der WSI-Mitteilungen möchte ich für verschiedene hilfreiche Einwände danken, auch wenn nicht allen Vorschlägen gefolgt wurde.
}

\footnotetext{
Rainer Trinczek, Prof. Dr., lehrt und forscht am Institut für Soziologie der Universität Erlangen-Nürnberg. Arbeitsschwerpunkte: Arbeits-, Organisations- und Managementsoziologie, Industrial Relations, Arbeitszeitforschung, Arbeit und Leben. e-mail: rainer.trinczek@soziol.phil. uni-erlangen.de
} 
wurde selbst, wie seit der Finanzkrise ab 2008 unschwer zu erkennen ist, zur Ursache häufig scheinbar erratischer Verwerfungen in der Real-Ökonomie, die nur bedingt kalkulierbar erscheinen.

Neben dieser generellen Verunsicherung verschärfen gestiegene Mobilität von Kapital sowie die dadurch ermöglichten Verlagerungsandrohungen von Unternehmen die Machtasymmetrie zuungunsten der Beschäftigten: Der angedrohte Verlust des Arbeitsplatzes stellt bekanntlich das sogenannte Totschlag-Argument in innerbetrieblichen Politikprozessen dar, das für maximale Erpressbarkeit von Belegschaften und ihrer Interessenvertretungen sorgt.

\subsection{VERÄNDERTE STRUKTUR DER ÖKONOMIE}

Mit welchen Begriffen man die aktuelle Gesellschaft auch immer belegen mag - ob postindustrielle Dienstleistungsgesellschaft, Wissensgesellschaft oder Kommunikationsgesellschaft -, stets ist damit die Beobachtung eines erheblichen Bedeutungsverlustes des Verarbeitenden Gewerbes verbunden, und zwar sowohl in quantitativer als auch symbolischer Hinsicht. Industriearbeit stellte bis in die 1970er Jahre hinein den paradigmatischen Bezugspunkt der öffentlichen und wissenschaftlichen Beobachtung von Arbeit dar, mit dem Facharbeiter als zentraler Figur. Dieser (männliche) Facharbeiter galt gewissermaßen als der idealtypische Beschäftigte der deutschen Nachkriegsökonomie. Auch wenn Facharbeiter faktisch nie die stärkste Beschäftigtengruppe unter den Beschäftigten darstellten, so war ihre hervorgehobene Stellung doch der zeitweisen Dominanz des Verarbeitenden Gewerbes innerhalb der bundesdeutschen Wirtschaft geschuldet sowie der Tatsache, dass gewerbliche Beschäftigte (aber eben auch Un- und Angelernte!) die größte Statusgruppe unter den abhängig Erwerbstätigen waren.

Dies hat sich dramatisch geändert: Würde man heute nach einer ähnlichen symbolischen Repräsentanz der aktuellen Ökonomie suchen, würde man eher auf akademisch gebildete, hochqualifizierte Büroarbeiter oder "Symbolanalytiker“ (wie Robert Reich sie genannt hat) stoßen.

Was sich hier widerspiegelt, ist zum einen eine Verschiebung der Wirtschaftssektoren hin zum tertiären Sektor, dem
2008 bereits $73 \%$ der Erwerbstätigen zuzuordnen waren, zum anderen das überproportionale Wachstum hochqualifizierter Beschäftigung im (expandierenden) tertiären Sektor (vgl. Schmidt 2010).

Dies bedeutet freilich nicht, dass nicht auch Wirtschaftszweige mit dominant unund angelerntem Personal expandieren würden; zu denken ist hier u. a. an die Systemgastronomie, die filialisierten Discounter, den Logistiksektor, das Sicherheits- und Reinigungsgewerbe sowie die haushaltsnahen Dienstleistungen. Nicht umsonst lässt sich in Deutschland seit Mitte der 1990er (mit einer gewissen Stagnation seit 2006) eine beachtliche Ausweitung des sogenannten Niedriglohnsektors beobachten: Legt man das Kriterium der Organisation für wirtschaftliche Zusammenarbeit und Entwicklung (OECD) an, dass als Niedriglohnbeschäftigter jemand zu gelten habe, der unterhalb von zwei Dritteln des medianen Stundenlohns verdient, betrug der Anteil der Niedriglohnbeschäftigten unter den abhängig Beschäftigten 2008 über 20\%; betroffen sind also über 6,5 Mio. Beschäftigte (Kalina/Weinkopf 2010).

Im Saldo überwiegt jedoch im wirtschaftlichen Strukturwandel der zunehmende Bedarf an hohen und mittleren Qualifikationen im Rahmen des wirtschaftlichen Strukturwandels - und dies nicht nur in Deutschland, sondern offensichtlich (zumindest) in ganz Europa (CEDEFOP 2008).

\subsection{DEMOGRAFISCHER WANDEL UND ERWERBSTÄTIGENSTRUKTUR}

Dass sich im Zuge des demografischen Wandels die Struktur der Erwerbstätigen nachhaltig verändern wird, steht außer Frage. Nicht nur, dass nach der 12. Koordinierten Bevölkerungsvorausberechnung des Statistischen Bundesamtes das Erwerbspersonenpotenzial zwischen 2008 und 2060 um - je nach Zuwanderungsszenario $-28 \%$ bis $34 \%$ schrumpfen wird (Destatis 2009a; vgl. auch für detaillierte Analysen bis 2030: Statistische Ämter des Bundes und der Länder 2009), sondern es werden gleichzeitig in allen Szenarien drei absehbare Veränderungen in der Erwerbstätigenstruktur beschrieben: Die Erwerbsquoten von Frauen und Älteren werden zunehmen und der Anteil von Personen mit Migrationshintergrund am Erwerbspersonenpotenzial wird ansteigen. Alle drei Entwicklungsprozesse werden in den einschlägigen Veröffentlichungen als weitgehend alternativlos gehandelt.

Angesichts des schrumpfenden Erwerbspersonenpotenzials wird die verstärkte Arbeitsmarkt-Mobilisierung von Frauen als nach wie vor großer Beschäftigungsreserve als zwingend angesehen. Die gilt insbesondere auch, weil Frauen bei den jüngeren Alterskohorten aufgrund des veränderten Bildungsverhaltens bzw. -erfolgs der Geschlechter ohnehin auf dem Weg sind, die Mehrheit der Hochqualifizierten in Deutschland zu stellen, was die Nutzung dieses Human-Ressourcen-Potenzials umso dringlicher erscheinen lässt; allerdings ist noch immer ein hochgradig selektives geschlechtsspezifisches Auswahlverhalten hinsichtlich der gewählten Studiengänge zu beobachten, wobei allerdings ein gewisses Aufholen bei Studienabschlüssen in den von Frauen bislang eher seltener gewählten Bereichen Mathematik und Naturwissenschaften (von $38 \%$ im Jahr 2000 auf $41 \%$ 2008) und Ingenieurwissenschaften (2000: 20\%; 2008: $23 \%$ ) festzustellen ist (BMFSFJ 2011).

Da gleichzeitig auch das Interesse von Frauen an einer Erwerbstätigkeit - wohl nicht zuletzt vermittelt über die zunehmend höheren Qualifikationen - bis heute zunimmt, dürfte eine weiterhin steigende Erwerbsquote von Frauen gesichert sein. Getrübt wird dieses Bild in den Augen mancher Arbeitsmarktpolitiker lediglich durch den hohen (und wachsenden) Anteil an Teilzeitbeschäftigung von Frauen (2000: 37,7\%; 2009: 44,8\%), was zweifelsohne in den lediglich „,rhetorisch modernisierten“ Geschlechterverhältnissen (Wetterer 2003), also den faktisch nach wie vor weitgehend traditionellen Modi privater geschlechtlicher Arbeitsteilung, seine Ursachen hat.

Festzuhalten bleibt jedoch, dass über das aus ganz unterschiedlichen Quellen (von der Bildungsökonomie über die Arbeitsmarkt- und Sozialpolitik bis hin zur Frauenbewegung) gespeiste und breit geteilte Interesse an einer zunehmenden weiblichen (Vollzeit-)Erwerbstätigkeit neue Themen auf die (arbeits-)politische Agenda gekommen sind: Von der viel zitierten Work-Life-Balance über flexiblere Arbeitszeitmodelle bis hin zu solch handfesten Themen wie Kinderkrippenplätze oder betriebseigenen Kinderbetreuungseinrichtungen, was den Eltern eine flexiblere Kombination von Arbeit und Familie erlaubt und damit gleichzeitig einer ge- 
wissen Entgrenzung von Betriebs- und Haushaltssphäre Vorschub leistet.

Neben der erhöhten weiblichen Erwerbstätigkeit steht die zunehmende Erwerbstätigkeit älterer Arbeitnehmerinnen und Arbeitnehmer gleichfalls auf der Tagesordnung der Politik. In diese Richtung drängen verschiedene Interessen: So sehen Sozialpolitiker in einem längeren Erwerbsleben und einem hinausgeschobenen Renteneintrittsalter eine gute Chance, die vom demografischen Wandel arg belasteten Sozialversicherungssysteme ein Stück weit zu entlasten; Arbeitsmarktpolitiker schätzen ältere Beschäftigte als Reserve, um den absehbaren Rückgang des Erwerbspersonenpotenzials etwas abzupuffern; Unternehmen entdecken den hohen Wert des aufgeschatzten Erfahrungswissens älterer Mitarbeiterinnen und Mitarbeiter sowie die Sinnhaftigkeit altersgemischter Teams (auch wenn die Wissenschaft da etwas skeptischere Befunde produziert; vgl. Becker 2008), und die Weiterbildungsindustrie erhofft sich von den dann notwendigerweise verstärkten Weiterbildungsanstrengungen der Betriebe für ihre älteren Beschäftigten (life-long-learning) ebenfalls einen gewissen Aufschwung.

Auch über die Zunahme der Beschäftigung Älterer werden neue arbeitspolitische Fragen thematisiert bzw. alte arbeitspolitische Themen neu auf die Tagesordnung gesetzt: Dies betrifft insbesondere Themen wie Arbeitsintensität (die nicht so hoch sein darf, dass sie ein längeres Berufsleben unmöglich macht), Ergonomie (mit Blick auf altersgerechte Arbeitsplätze), Health \& Safety und das bereits erwähnte Weiterbildungsthema.

Obwohl die öffentliche Migrationsdebatte nach wie vor zwischen Xenophobie und einem positiven Multikulturalismus schwankt, sind sich die Arbeitsmarktexperten einig, dass ein positiver Migrationssaldo zwingend ist, um das Erwerbspersonenpotenzial durch den demografischen Wandel nicht bedrohlich absinken zu lassen. Daher ist weniger strittig, dass Zuwanderung gefördert werden soll - zumal der Wanderungssaldo in Deutschland 2008 und 2009 negativ war und auch seit einigen Jahren nur ausnahmsweise in die Nähe der in den unterschiedlichen demografischen Szenarien des Statistischen Bundesamtes hinterlegten Größen gelangt ist (Bundesamt für Migration und Flüchtlinge 2011). Ebenso unstrittig wie die schiere Erhöhung des Wanderungssaldo scheint es zu sein, dass insbesondere die Zuwanderung Hochqualifizierter gefördert werden soll, und das vor allem in Bereichen, wo man die schwerwiegendsten Knappheitsphänomene auf den (Teil-)Arbeitsmärkten befürchtet. Nach wie vor unklar scheint allerdings die Strategie zu sein, mit der man dieses Ziel erreichen möchte. Deswegen ist die Annahme nicht unplausibel, dass Zuwanderung in Zukunft weiterhin (nach offiziösen Standards) eher niedrigqualifizierte Zuwanderung sein wird, was die bereits erkennbaren Probleme einer sogenannten qualifikatorischen Unterschichtung von Belegschaften mit den entsprechenden Integrationsproblemen eher verfestigen dürfte.

Insgesamt ist unter den einschlägigen Experten jedoch unstrittig: Belegschaften werden in Zukunft weiblicher, älter und multikultureller. Dies bedeutet, dass sich (männliche wie weibliche) Beschäftigte nicht nur darauf einstellen müssen, zukünftig bis in ein höheres Alter und in einem multikulturelleren Kontext als heute $\mathrm{zu}$ arbeiten; gleichzeitig dürfte angesichts zunehmender weiblicher Erwerbstätigkeit auch der Druck auf ein Rearrangement privater Formen der Lebensführung und tradierter Modi geschlechtsspezifischer Arbeitsteilung weiter zunehmen.

\subsection{VON WELFARE ZU WORKFARE}

Die veränderte arbeitspolitische Regulierung von Arbeit wird seit einigen Jahren verstärkt als grundlegender Wechsel des sozialpolitischen Regimes diskutiert, der schlagwortartig als Wechsel „von Welfare zu Workfare" bezeichnet wird. Gemeint ist damit im Wesentlichen, dass - gemäß dem Motto „Fördern und Fordern“ - Ansprüche auf sozialpolitische Leistungen verstärkt an die Erfüllung von Pflichten durch die potenziellen Leistungsempfänger geknüpft werden. Diese häufig mit dem Begriff der Aktivierung umschriebenen institutionellen Erwartungen beziehen sich vor allem auf die verstärkte und teilweise auch eigeninitiative Bereitschaft, sich eine neue Arbeitsstelle zu suchen bzw. die eigene Beschäftigungsfähigkeit (employability) durch Weiterqualifizierung und die Nutzung von Beschäftigungsmaßnahmen zu verbessern. Gleichzeitig werden die Leistungen restriktiver vergeben (etwa Erhöhung von Anspruchsvoraussetzungen, Befristungen der Anspruchsdauer etc.); die Verschärfung der Zumutbarkeitsanforde- rungen zwingt Arbeitslose ggf. in schlecht bezahlte Beschäftigungsverhältnisse, die eigentlich weder der Qualifikation noch dem sogenannten Marktwert der Betroffenen entsprechen. Und gleichzeitig wird der Sanktionsapparat für den Fall mangelnder Fügsamkeit ausgebaut, sodass solche Beschäftigungsuchenden, die behördenseitig als nicht hinreichend kooperativ eingestuft werden, dann auch erheblichen Sanktionen unterworfen werden können (vgl. unter anderen Blank 2011; Brütt 2011).

Ohne hier detailliert auf die diversen Einzelmaßnahmen einzugehen, lässt sich insgesamt festhalten, dass durch vielfältige Neuausrichtungen in der Sozial- und Beschäftigungspolitik die für den traditionellen Wohlfahrtsstaat konstitutive (Teil-) De-Kommodifizierung von Arbeitskraft erheblich zurückgeschraubt wurde und damit Beschäftigte mit ihrem Arbeitsvermögen heute in stärkerem Maße direkt den Zwängen des Arbeitsmarktes ausgesetzt sind, als dies bis in die 1980er Jahre hinein der Fall war.

\subsection{SEGMENTIERUNG DER ÖKONOMIE IN VERSCHIEDENE MITBESTIMMUNGS- UND PARTIZIPA- TIONSKONSTELLATIONEN}

Die Industrial-Relations-Forschung hat sich in den letzten Jahren verstärkt der Tatsache geöffnet, dass der für Deutschland als typisch geltende Modus der Interessenvertretung, also das duale System in der Kombination aus Betriebsrat und Flächentarifvertrag, empirisch nicht mehr dasjenige Modell ist, in dessen Rahmen die Arbeitsverhältnisse der Mehrheit der abhängig Beschäftigten in Deutschland reguliert werden. Wie Ellguth/Kohaut (2011) bei ihrer jüngsten Auswertung des IABBetriebspanels herausstreichen, ist „nur eine Minderheit der in der Privatwirtschaft (ab fünf Beschäftigte) beschäftigten Arbeitnehmer in Betrieben tätig, die zur Kernzone des dualen Systems der Interessenvertretung zu zählen sind. Diese Zone umfasst ein knappes Drittel der Beschäftigten in Westdeutschland und weniger als ein Fünftel in Ostdeutschland“ (ebd. S. 247).

Dies bedeutet, dass sich in Deutschland eine vielfältig ausdifferenzierte Landschaft an unterschiedlichen Formen der Interessenvertretung herausgebildet hat (vgl. etwa Artus et al. 2006; Hauser-Ditz et al. 2008). Die einschlägigen Ergebnisse lassen sich in der These von einer Pluralisierung und 
Segmentierung der Arbeitsbeziehungen angemessen fassen.

Nicht nur, dass wir mit einer äußeren und inneren Erosion des Flächentarifvertrags konfrontiert sind, gleichzeitig ist hinsichtlich der betrieblichen Interessenvertretung seit den 1990er Jahren eben auch eine quasi neue Welt jenseits der betriebsverfassungsrechtlichen Normen sukzessiv entdeckt worden, die in sich wiederum erheblich differenziert ist: Das Spektrum reicht dabei von neuen Sweatshops bis hin zu modernen Wissensarbeitsplätzen mit (mehr oder weniger) aufgeklärten HumanResource-Management (HRM)-Politiken.

Das Modell der institutionell abgesicherten kooperativen Konfliktverarbeitung zwischen Betriebsrat und Management im Rahmen des dualen Modells findet sich gegenwärtig wesentlich nur noch in den (schrumpfenden!) Kernbereichen des Verarbeitenden Gewerbes und im Öffentlichen Dienst - aber es hat nachhaltig an stilbildender Kraft für die Arbeitsbeziehungen in Deutschland insgesamt eingebüßt.

Dies bedeutet für Beschäftigte, dass sie sich im Kontext einer unübersichtlich gewordenen Mitbestimmungslandschaft bewegen müssen, in der nur teilweise die gesetzlich vorgesehenen Formen der Interessenvertretung vorfindlich sind: Teilhabe bedeutet unter diesen Umständen häufig Eigeninteressenvertretung - was je nach betrieblichem Kontext und individueller Arbeitsmarktmacht mitunter erfolgversprechend ist, häufig jedoch auf eine Schwächung der Position der Beschäftigten hinausläuft.

\section{Zum Wandel betrieblicher Arbeitskonstellationen}

Während im letzten Abschnitt veränderte Kontextfaktoren von Arbeit behandelt wurden, geht es nun um den Wandel konkreter Arbeit. Dabei verbietet sich das Formulieren schlanker Thesen, da die Realität konkreter Arbeitssituationen so stark ausdifferenziert ist, dass sich gleichzeitig diametral entgegengesetzte Entwicklungen beobachten lassen: Zunehmende Autonomie in der Arbeit auf der einen Seite und Re-Taylorisierung auf der anderen, hohe Bonuszahlungen hier und Prekarisierung dort. Konnte Ulrich Beck noch von einem sozialstruk- turellen „Fahrstuhleffekt“ sprechen, der auch die Deprivilegierten der Gesellschaft in der Nachkriegszeit von der allgemeinen Wohlfahrtssteigerung profitieren ließ (Beck 1986), so scheint diese Phase definitiv beendet zu sein: Während sich die Arbeits- und Lebenssituation für manche verbessert, hat sie sich für andere definitiv verschlechtert. ${ }^{2}$

Vor diesem Hintergrund sollen im Folgenden einige Entwicklungsstränge von Arbeit skizziert werden, die aus den besagten Gründen freilich keine allgemeinen Trends abbilden, sondern sich selektiv für bestimmte Beschäftigtengruppen als relevanter darstellen als für andere; dabei handelt es sich um:

\section{- Destandardisierung (3.1), \\ - Prekarisierung (3.2), \\ - Subjektivierung (3.3), \\ - Intensivierung (3.4), \\ - Flexibilisierung (3.5), \\ - Entgrenzung (3.6).}

Dass diese Prozesse vielfach miteinander verwoben sind, versteht sich von selbst auch wenn diese Zusammenhänge hier nicht en detail ausgeführt werden können.

\subsection{DESTANDARDISIERUNG VON ARBEIT}

Destandardisierungsprozesse von Arbeit werden in der einschlägigen Forschung mit Blick auf verschiedene Felder thematisiert; so wird etwa seit geraumer Zeit darüber debattiert, ob die für das deutsche Arbeitssystem so typische Orientierung an Berufen (als standardisierter Definition von Kenntnissen, Fähigkeiten und Fertigkeiten) angesichts veränderter arbeitsorganisatorischer Kontexte noch zukunftsfähig sei (vgl. etwa Baethge/Baethge-Kinsky 1998). Oder es wird über die Destandardisierung und Individualisierung von (Erwerbs-)Biografien im Übergang zur Zweiten Moderne diskutiert.

Beherrscht wird die Debatte jedoch eindeutig von der Frage der Destandardisierung von Beschäftigungsverhältnissen. Dass sich diese gegenwärtig als erheblich pluraler darstellen als früher und dass das Normalarbeitsverhältnis daher in gewisser Hinsicht erodiert, steht heute außer Frage; ggf. kontrovers diskutiert werden Ausmaß und Geschwindigkeit dieses Prozesses. Differenzen in den quantitativen Berechnungen sind dabei wesentlich auf die verwendeten Definitionen dessen, was als Normalarbeitsverhältnis gilt, und die genutzten Datenquellen zurückzuführen: So errechnet beispielsweise das Statistische Bundesamt für das Jahr 2008 auf der Grundlage des Mikrozensus und einer engeren Definition hinsichtlich der Teilzeitarbeit eine Quote von gut 25\% atypischer Arbeitsverhältnisse unter den abhängig Beschäftigten (Destatis 2009b), während Brehmer/Seifert (2008) auf der Grundlage des Sozio-oekonomischen Panels (SOEP) und einer breiteren Berücksichtigung von Teilzeitarbeit in einer begründeten Schätzung für das Jahr 2007 bereits auf $37 \%$ kommen. Einigkeit herrscht jedoch darüber, dass die sogenannten atypischen Beschäftigungsformen (Teilzeitarbeit, geringfügige Beschäftigung, befristete Arbeitsverhältnisse und Leiharbeit) insgesamt seit Mitte der 1990er Jahre vergleichsweise kontinuierlich zunehmen, wobei es durchaus erhebliche Schwankungen im Einzelfall geben kann, wie dies etwa bei der konjunktursensiblen Leiharbeit während der letzten Krise zu beobachten war. Dass zusätzlich auch unter der anwachsenden Zahl an Solo-Selbstständigen, die das Statistische Bundesamt für das Jahr 2008 mit 2,1 Mio. auswies (Destatis 2009b), in erheblichem Umfang schein-selbstständige Beschäftigungsformen zu finden sein dürften, erhöht das Ausmaß atypischer Beschäftigung zusätzlich. Kurzum: Beschäftigte, die auf dem Arbeitsmarkt eine neue Stelle suchen, müssen mit einer vergleichsweise hohen Wahrscheinlichkeit damit rechnen, atypisch beschäftigt $\mathrm{zu}$ werden.

Es sind freilich weniger Fragen des quantitativen Umfangs atypischer Beschäftigung, die die Diskussion beherrschen, als vielmehr die Frage der Folgen, die dies für die betroffenen Beschäftigten hat. Dabei ist klar, dass nicht jeder atypisch Beschäftigte gleich unter problematischen Arbeits- und Entlohnungsbedingungen zu leiden hat. Gleichzeitig ist auch klar, dass die Destandardisierung der Beschäftigungsverhältnisse erheblich zur zunehmenden Prekarisierung von Arbeit beigetragen hat.

\footnotetext{
2 So u. a. auch bereits Butterwege (2006), der dafür allerdings das etwas schiefe Bild des „Paternoster-Effekts" bemühte; jeder der die Funktionsweise von Paternostern kennt, weiß, dass diese an den beiden Endpunkten umdrehen: Wer also ganz oben angekommen ist, fährt automatisch wieder ganz runter et vice versa. Dies entspricht nun freilich gerade nicht der Realität.
} 


\subsection{PREKARISIERUNG VON ARBEIT}

Der Prekaritätsdiskurs hat Konjunktur, die Anzahl der Veröffentlichungen mit Bezug auf Prekarität hat unübersehbare Ausmaße angenommen. Dies dürfte nicht zuletzt der Tatsache geschuldet sein, dass Prekarität ein vergleichsweise unbestimmter Begriff ist, der vielfältig definiert und damit auch vielfältig einsetzbar ist (vgl. auch Kraemer 2008). Dabei wird von manchen versucht, Prekarität „hart“ zu operationalisieren (etwa Brehmer/Seifert 2008): über Einkommensniveau, Beschäftigungsstabilität, Beschäftigungsfähigkeit und Einbindung in das System sozialer Sicherung, während andere den handlungsrelevanten Aspekt subjektiv gefühlter Prekarität stärker betonen (etwa Brinkmann et al. 2006). Und wieder andere verweisen auf die Notwendigkeit der Ausweitung der Prekarisierungsdebatte auf den ,gesamten Lebenszusammenhang" (Jürgens 2011).

Ohne hier auf die Details der Debatte eingehen zu können, kann doch als Quintessenz zusammengefasst werden, dass es in der Tat eine erhebliche Ausweitung von prekären Beschäftigungsverhältnissen gegeben hat, die auch weit in bislang als eher ungefährdet eingestufte Bereiche akademischer Tätigkeiten (Generation Praktikum) hineinreicht; im Kern dieser Debatte steht die Zunahme schlecht bezahlter und unsicherer Beschäftigung, was unter Stichworten wie Niedriglohnsektor, working poor und Aufstocker bearbeitet wird. Ohne Zweifel ist in Deutschland die Expansion eines Niedriglohnsektors politisch betrieben und im intendierten Sinne dann auch erfolgreich durchgesetzt worden; es wird davon ausgegangen, dass kein anderes Land der Europäischen Union (EU) einen vergleichbar großen Niedriglohnsektor hat wie Deutschland. „Die Niedriglohnschwelle von 2/3 des jeweiligen Medianentgelts wird im Jahr 2009 in Westdeutschland von $20,2 \%$ und in Ostdeutschland von 21,3\% der Vollzeitbeschäftigten unterschritten“, berichtet die Bundesagentur für Arbeit (BA) (2010). Nach Auswertungen des Instituts für Arbeitsmarkt- und Berufsforschung (IAB) gibt es rund 1 Mio. abhängig Beschäftigte, die in sogenannten Aufstocker-Bedarfsgemeinschaften wohnen, darunter gut $20 \%$ Vollzeitbeschäftigte, die trotz Vollerwerbstätigkeit kein Einkommen über dem Hartz-IV-Niveau erzielen können und deren Einkommen deswegen von der BA auf Hartz IV aufgestockt wird. Im arith- metischen Mittel verdienen Aufstocker in Westdeutschland brutto $€ 7,00 /$ Stunde, in Ostdeutschland $€ 6,02$ (alle Zahlen aus Dietz et al. 2009). Vollerwerbstätigkeit allein kann unter diesen Umständen kein Absinken in Armut mehr verhindern.

Gleichzeitig stimmt aber sicherlich auch, dass sich ein erhöhtes subjektives Verunsicherungspotenzial, eine gefühlte Prekarisierung, in den letzten Jahren weit über die Prekarier im engeren Sinne hinaus bis in die sogenannten Mittelschichten hinein ausgebreitet hat. Ob es jedoch Sinn macht, diese teils recht diffusen Ängste vor dem sozialen Abstieg gemeinsam mit den realen Existenzsorgen der working poor unter den Begriff der Prekarisierung zu diskutieren, mag bezweifelt werden. Gleichwohl ist es sicher richtig, dass der wachsende Bereich prekärer, unsicherer und schlecht bezahlter Arbeit Ausstrahleffekte in die (noch) stabilen Segmente hinein hat.

\subsection{SUBJEKTIVIERUNG VON ARBEIT}

Ähnlich wie der Begriff der Prekarisierung wesentlich mit Blick auf das untere Ende des Statusgefüges von Arbeit diskutiert wird, zielt der Begriff der Subjektivierung von Arbeit auf veränderte Arbeitssituationen vor allem am oberen Ende des Qualifikationsspektrums. In der Subjektivierungsdebatte wird vor allem auf die doppelte Stoßrichtung von Subjektvierung verwiesen: „Die Individuen tragen mehr ,Subjektives ' in die Arbeit hinein und/oder die Arbeit fordert mehr ,Subjektives' von den Individuen“ (Kleemann et al. 1999, S. 2). Dies verweist auf einen gewissen Wechsel in der Kontrollperspektive von Unternehmen gegenüber ihren (hoch-)qualifizierten Beschäftigten: Während früher vom Management vielfach befürchtet wurde, Beschäftigte würden Freiräume, die ihnen in der Arbeit eingeräumt werden, eigensinnig, d.h. nicht notwendigerweise zum Nutzen und Wohle des Unternehmens nutzen, hat sich diese Einschätzung heute vielerorts geändert. In diesem Kontext wurde dann häufig ein Kontrollregime etabliert, das sich im Rahmen eines „Management by objectives" auf das Aushandeln einer Zielvorgabe beschränkt und dann den Prozess der Zielerreichung weitgehend der autonomen Gestaltung durch die Beschäftigten überlässt. Damit sind diese gefordert, Selbstorganisationsfähigkeiten in den Arbeitsprozess einzubringen und ihr
Projekt quasi wie kleine Intrapreneure zu managen (vgl. in diesem Kontext auch die These vom "Arbeitskraftunternehmer“, Voß/Pongratz 1998).

Das interessante an der Subjektivierungsdebatte war nun, dass festgestellt werden konnte, dass diese neuen Anforderungen von manchen Beschäftigten gerne angenommen werden, weil sie selbst ein ausgeprägtes Interesse an der Selbstgestaltung ihrer Arbeit aufweisen, arbeitsinhaltlich stark intrinsisch motiviert sind und traditionellen bürokratischhierarchischen Anordnungssystemen eher skeptisch gegenüberstehen. Allerdings wurde ebenso rasch klar, dass Beschäftigte in solchen Konstellationen häufig zur Selbst-Ausbeutung (wesentlich in Form überlanger Arbeitszeiten) neigen, was wiederum erheblichen Anpassungsdruck auf die weniger „subjektivierten“ Kolleginnen und Kollegen ausübt, zumal wenn man gemeinsam in Projekten arbeitet. „Arbeiten ohne Ende“ scheint der Slogan zu sein, der die Situation in diesen neuen, vermeintlich freien Arbeitskonstellationen am deutlichsten beschreibt.

Während ein verstärktes subjektiv-expressives Verhältnis zur Arbeit offensichtlich vermehrt auch im mittleren Qualifikationsspektrum vorzufinden ist (hierzu bereits Baethge 1991) scheint es so zu sein, dass die Ansprüche einer weitgehend uneingeschränkten Verfügbarkeit für das Unternehmen wesentlich im oberen Qualifikationsbereich akademisch ausgebildeter Beschäftigter angenommen werden - und dies umso freiwilliger, je klarer man sich noch auf einem intakten Aufstiegs- und Karrierepfad wähnt.

\subsection{INTENSIVIERUNG VON ARBEIT}

Intensivierung von Arbeit ist seit den frühesten arbeits- und industriesoziologischen Studien der Nachkriegszeit ein regelmäßig erhobener Befund. Immer wieder wurde davon berichtet, dass die Leistungsschraube, verglichen mit früher, angezogen worden sei. Während im gewerblichen Bereich über den verschärften Akkord Beschwerde geführt wurde, verwiesen Angestellte auf erhöhte Arbeitslast in gleicher Arbeitszeit. Regelmäßig wurde der Verweis auf die Intensivierung der Arbeit durch Managementvertreter mit dem Hinweis gekontert, es sei „noch Luft" in den Prozessen gewesen bzw. technische oder organisatorische Rationalisie- 
rungsmaßnahmen hätten es erlaubt, eine erhöhte Leistung von den Beschäftigten $\mathrm{zu}$ fordern. Beliebt war auch das probate empirische Argument, es scheine doch zu funktionieren - die Beschäftigten würden das neue Pensum ja schaffen, ohne dass dies zu spürbaren Ausfällen bzw. zu erhöhten Krankmeldungen geführt hätte. Dabei wird freilich gerne übersehen, in welchem Umfang Beschäftigte seit Längerem bereits kompensatorisch dem zunehmenden Arbeitsstress in der außerbetrieblichen Sphäre entgegensteuern: mehr Sport, gesünderes Leben, Entspannungstrainings etc.

Nun erhöhen sich in den letzten Jahren jedoch die Hinweise darauf, dass die Arbeitsintensität und der daraus resultierende arbeitsbedingte Stress zumindest in Teilbereichen ein gewisses Grenzniveau überschritten haben, was sich auch spürbar in quantitativen Größen niederschlägt; der aktuelle öffentliche Diskurs über burnout als Volkskrankheit ist hierfür symptomatisch. Und in der Tat belegen Studien, die sich mit arbeitsbedingten Ursachen für das Auftreten von depressiven Störungen beschäftigten, den Zusammenhang zwischen steigender Arbeitsintensität und psychischen Störungen: „Je höher die objektiv bewertete Arbeitsintensität war, desto häufiger trat Major Depression und Depressivität auf“ (Rau et al. 2010, S. 7).

Gleichzeitig berichten die Krankenkassen schon seit einiger Zeit über ansteigende Zahlen psychisch bedingter Fehlzeiten in Betrieben. So schreibt etwa die DAK in ihrem Gesundheitsreport 2011 unter der Überschrift „Betriebliche Einflussfaktoren auf den Krankenstand“ nüchtern: „Viele Dienstleistungsunternehmen einschließlich der öffentlichen Verwaltungen stehen verstärkt unter Wettbewerbsdruck bei fortschreitender Verknappung der Ressourcen. In der Folge kommt es zu Arbeitsverdichtungen und , Rationalisierungen' und vielfach auch zu Personalabbau. Daraus können belastende und krank machende Arbeitsbelastungen (z. B. Stressbelastungen) entstehen, die zu einem Anstieg des Krankenstandes führen“ (DAK 2011). Und auch das wissenschaftliche Institut der Allgemeinen Ortskrankenkasse (AOK) meldet im April 2011 unter der Überschrift „Burnout auf dem Vormarsch “: „Insbesondere psychische Erkrankungen sind weiterhin auf dem Vormarsch. Die Fehlzeiten aufgrund psychischer Erkrankungen sind seit 1999 um nahezu 80 Prozent angestiegen und führen zu langen Ausfallzeiten:
Diese dauern mit 23,4 Tagen je Fall doppelt so lange wie der Durchschnitt mit 11,6 Tagen je Fall im Jahr 2010“ (WIdO 2011).

Dies scheinen ernsthafte Hinweise darauf zu sein, dass sich Betriebe hinsichtlich der Intensivierung von Arbeit insofern auf eine Art Scheitelpunkt hinbewegen, als Beschäftigte in spürbarem Umfang dem angestiegenen Leistungsdruck nicht mehr gewachsen sind - ein Befund, der angesichts der Pläne zur Verlängerung der Lebensarbeitszeit im Kontext der Debatte um den demografischen Wandel zusätzlich an Dramatik gewinnt. Mit den erhöhten Fehlzeiten werden die Folgen der Arbeitsintensivierung für Unternehmen allerdings nun auch in einem Modus spürbar, für den sie prinzipiell gut empfänglich sind - Geld. Es bleibt abzuwarten, ob und inwiefern Unternehmen reagieren, wenn nach ihren Kriterien relevante Zusatzkosten entstehen.

\subsection{FLEXIBILISIERUNG VON ARBEIT}

Die Durchsetzung eines „flexibel-marktzentrierten Produktionsmodells" (Dörre 2003) ist gekennzeichnet nicht nur durch beständige Reorganisationsbemühungen von Unternehmen, die häufig darauf abzielen, stärker als bisher Wettbewerbselemente und Marktprinzipien in die Binnenstrukturen von Unternehmen zu integrieren, also Betriebe auch in ihrem Innenverhältnis über Marktprozesse zu steuern (Sauer 2010; vgl. hierzu auch die interessante Studie von Binswanger 2010). Auf diese Weise werden Cost- oder ProfitCenter-Strukturen geschaffen oder Betriebsteile auch gleich real unternehmensrechtlich zu selbstständigen Einheiten transformiert, wodurch der Marktdruck näher an die Organisation herangeführt werden soll. Die Reaktionen sind bekannt: Verknappung von Material-, Personal- und Zeitpuffern, Aufbau flexibler Randbelegschaften bzw. von Beschäftigungspuffern über die verstärkte Nutzung von Leiharbeit als „komplementärem Flexibilisierungsinstrument" (Promberger 2006) und insbesondere die Ausweitung flexibler Arbeitszeiten im Rahmen diverser Kontenregelungen. Über den Auf- und Abbau der entsprechenden Zeitguthaben können die Flexibilitätsanforderungen des Marktes von den Betrieben direkt an die Beschäftigten weitergereicht werden; so wird etwa die Tatsache, dass die Finanz- und Wirtschaftskrise 2008/09 in vergleichsweise geringem Ausmaß auf den Arbeitsmarkt durchgeschlagen hat, u. a. auch mit dem massiven Abbau von Zeitguthaben der Beschäftigten auf den entsprechenden Zeitkonten begründet (etwa Möller 2010). Dies scheint alte Zeitkonflikte neu aufzulegen (Groß/Seifert 2010), in denen es seit Beginn der Flexibilisierungsdebatte in den frühen 1980er Jahren stets um die Frage ging, wer im Konfliktfall letztlich die Verfügungsgewalt über Arbeitszeitentscheidungen im Betrieb hat: der Beschäftigte, mit seinem Anliegen, die Arbeitszeit souverän seinen Bedürfnissen anzupassen, oder das Management, dass in den Arbeitszeiten der Mitarbeiter vorrangig einen betrieblichen Flexibilitätspuffer sieht.

Gleichwohl nehmen sich Zeitkonflikte heute anders aus als früher, da es in den letzten 20 Jahren eine schleichende $\mathrm{Zu}$ nahme betrieblicher Verfügbarkeitsansprüche gegenüber ihren Beschäftigten gibt, die über moderne Informations- und Kommunikationsmedien forciert durchgesetzt werden können. So antworten in einer Umfrage des Bundesverbands der Betriebskrankenkassen $84 \%$ der Berufstätigen, dass sie außerhalb der regulären Arbeitszeiten für beruflichen Angelegenheiten (etwa für Arbeitgeber, Kollegen und Kunden) erreichbar seien, $51 \%$ sogar ,jederzeit", wobei allerdings Erreichbarkeit nur von $26 \%$ erwartet wird. Ein Großteil der Befragten scheint sich allerdings mit den Verfügbarkeitsansprüchen arrangiert zu haben, wobei jedoch die systematisch kritischeren Einschätzungen von denjenigen auffällig sind, die auf Erreichbarkeit verpflichtet sind: Während von den freiwillig Erreichbaren $66 \%$ antworten, dass sie die Erreichbarkeit außerhalb der Arbeitszeit „sehr wenig“ oder „überhaupt nicht" störe, trifft dies nur auf $41 \%$ derjenigen zu, von denen Erreichbarkeit erwartet wird (BKK 2010). Dass die Verfügbarkeit für arbeitsbezogene Belange auch jenseits der Arbeitszeit gleichzeitig eine zunehmende Entgrenzung von Arbeit und Privatleben bedeutet, versteht sich von selbst.

\subsection{ENTGRENZUNG VON ARBEIT}

Der Entgrenzungsbegriff wird regelmäßig als Passepartout für verschiedenste Phänomene genutzt, die als Erosionsprozesse von Grenzen und Strukturen interpretiert werden können, welche sich in der (fordistischen) Nachkriegsphase des Kapitalismus als normal herausgebildet hatten (vgl. Kratzer/Sauer 2003). Darunter fallen Verände- 
rungen, die bereits angesprochen wurden, wie etwa die Entgrenzung von Leistungszumutungen im Rahmen subjektivierter Arbeitsverhältnisse oder die Verflüssigung der Grenzen von Arbeit und Privatleben im Kontext neuer Verfügbarkeitserwartungen (vgl. auch Gottschall/Voß 2003; Jurczyk et al. 2009). Darüber hinaus wird der Entgrenzungsbegriff jedoch auch genutzt, um auf die zunehmende Auflösung des Betriebes als räumliche und soziale Einheit hinzuweisen; einschlägige Stichworte sind: Profit-Centre, virtuelles Unternehmen, Telearbeit, Mobil-Arbeit, Leiharbeit. Dies verweist auf eine fluidere Organisationsform von Arbeit, die Beschäftigten in geringerem Umfang als früher klare Strukturierungen in ihrem Erwerbsleben bietet, was von manchen als eine positive Gestaltungsherausforderung gerne angenommen, von anderen jedoch als eine nicht unerhebliche Belastung wahrgenommen wird.

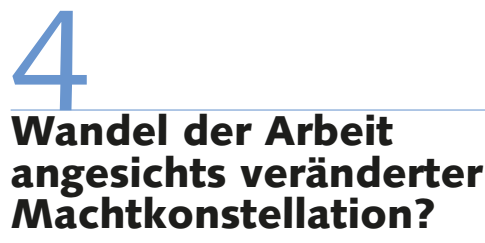

In den vorstehenden Abschnitten sind Veränderungstendenzen von Arbeit skizziert worden, die sich in den letzten Dekaden bereits durchgesetzt haben oder die sich -in aller Vorsicht - für die kommenden Jahre antizipieren lassen. Nun stellt sich der Wandel von Arbeit aber keineswegs als ein Automatismus oder Sachzwang dar, der sich hinter dem Rücken der Akteure vollzieht, sondern er ist Resultat eines arbeitspolitischen und damit machtgestützten Aushandlungsprozesses. Die beschriebenen Wandlungstendenzen können als Resultat eines Prozesses gesellschaftlicher Machtverschiebung rekonstruiert werden, der Mitte der 1970er Jahre einsetzt. Ohne dies hier näher ausführen zu können, lassen sich hierfür doch einige zentrale Stichworte benennen: Das Ende der Bretton-Woods-Ära, der damit verbundene Beginn der Liberalisierung der Finanzmärkte als Startpunkt der Globalisierung, der Übergang von keynesianischen zu neoliberal-monetaristischen Formen der Wirtschaftssteuerung und der Startpunkt einer lang anhaltenden Massenarbeitslosigkeit. Alle diese Faktoren haben zu einer erheblichen Machtverschiebung zugunsten des „Kapitals“ und zulasten von „Arbeit“ geführt; hinzu kommt, dass es den Unternehmen, ihren Verbänden und befreundeten Parteien gelungen ist, den öffentlichen Diskurs mit ihren Semantiken zunehmend zu dominieren: „Markt ist besser als Staat“; „Gewerkschaften sind die letzten Dinosaurier des Industriezeitalters“; „Leistung muss sich endlich wieder lohnen“; ,ein Arbeitsplatz ist ein hohes Gut, für das man als Beschäftigter mitunter auch etwas opfern muss" etc. pp.

Die Gewerkschaften hatten dieser Entwicklung wenig entgegenzusetzen: Ihre abnehmende Attraktivität bei den Beschäftigten (die sich seit den 1980er Jahren in sinkenden Mitgliederzahlen niedergeschlagen hat), das lockerer gewordene Verhältnis zur SPD (und damit der sinkende politische Einfluss ), das reduzierte Interesse des politischen Systems an einer korporatistischen Einbindung der Gewerkschaften und insbesondere die langjährige Massenarbeitslosigkeit haben die Macht der Gewerkschaften und betrieblichen Interessenvertretungen im Kern angegriffen: Die Entwicklung der Lohnquote und der Reallöhne der Beschäftigten in den letzten Dekaden können hierfür ebenso als Beleg herangezogen werden wie der flächendeckende Abbau übertariflicher (Sozial-) Leistungen in den Unternehmen.

Angesichts des demografischen Wandels könnte nun - ceterus paribus - damit gerechnet werden, dass die Arbeitslosigkeit wegen des erheblich reduzierten Angebots an Erwerbspersonen auf dem Arbeitsmarkt stark rückläufig wird und der Arbeitsmarkt eher von Knappheitsphänomenen gekennzeichnet sein dürfte; bereits heute wird vielfach von Fachkräftemangel gesprochen, der es Unternehmen erschwert, Beschäftigte mit den gewünschten FachQualifikationen auf dem Arbeitsmarkt zu rekrutieren. Dies dürfte - zumindest im oberen und mittleren Qualifikationssegment - in der Tat die Marktmacht der Beschäftigten (und damit möglicherweise auch der Gewerkschaften und Betriebsräte) wieder ansteigen lassen. Man könnte spekulieren, dass Beschäftigte dadurch verstärkt in die Lage versetzt werden, ihre eigenen Interessen und Ansprüche an Arbeit in höherem Maße gegenüber ihren Arbeitgebern durchzusetzen, als dies gegenwärtig der Fall ist; dies könnte auf eine Option zur Verbesserung von Arbeits- und Entlohnungsbedingungen verweisen.

Abgehängt von dieser Entwicklung dürften jedoch die niedrig qualifizierten An- und Ungelernten werden, da hier - nicht zuletzt über Migrationsprozesse wohl stets ein Überangebot an Beschäftigten zur Verfügung stehen wird. Es dürfte eine der wesentlichen zukünftigen Aufgaben von Gewerkschaften sein, der bereits sichtbaren Spaltung der Beschäftigten - in Gruppen mit vergleichsweise intakter individueller Arbeitsmarktmacht und solche ohne derartige Machtressourcen -entgegenzuarbeiten - es sei denn, Gewerkschaften möchten sich mit der Vertretung der „Beschäftigten-Aristokratie“ zufrieden geben. Der neu eingesetzte öffentliche Gerechtigkeitsdiskurs, der sich wesentlich an der wachsenden Armut in Deutschland auf der einen Seite und den exzessiven Gehältern mancher Manager und Banker auf der anderen Seite entzündet hat, könnte Gewerkschaften in einer stärker solidarischen Ausrichtung möglicherweise unterstützen.

Die entscheidende Frage wird jedoch sein, ob es tatsächlich zu den angedeuteten Knappheitsphänomenen auf dem Arbeitsmarkt mit dem entsprechenden Umschwung des Machtpendels zugunsten des Faktors Arbeit kommen wird. Unklar ist nämlich, wie sich die Nachfrageseite auf dem Arbeitsmarkt entwickeln wird. Dabei steht weniger die schon hinreichend komplexe Frage im Mittelpunkt, inwieweit sich eine schrumpfende Bevölkerung auf die gesamtgesellschaftliche Konsumnachfrage und damit auch auf die Nachfrage nach Arbeit auswirkt. Derzeit offen ist vielmehr, wie Unternehmen wohl auf die absehbare Verknappung des Arbeitskräfteangebots reagieren werden. In Zeiten der Globalisierung stehen den Betrieben mehr Optionen zur Verfügung als dies etwa während der Phase der Vollbeschäftigung in den 1960er und frühen 70er Jahren der Fall war. Die heute schon beobachtbaren Prozesse des Off-Shoring - auch im Hochqualifikationssegment (vgl. etwa für die IT-Industrie Boes/Kämpf 2011) - dürften erste Hinweise auf mögliche Reaktionsformen von Unternehmen auf die prognostizierten Veränderungen auf den Arbeitsmärkten sein.

Inwieweit also tatsächlich ein Umschwung in den gesellschaftlichen Machtverhältnissen zugunsten von Arbeit in den nächsten Jahren eintreten dürfte, darüber kann nur spekuliert werden. In gleicher Weise kann aber auch darüber spekuliert werden, ob das Modell eines flexiblen, „entfesselten" (Finanzmarkt-)Kapitalismus in sich ein nachhaltiges sein kann - oder ob es nicht zunehmend an individuelle wie gesellschaftliche Grenzen stößt. 
Artus, I./Böhm, S./Lücking, St./Trinczek, R. (Hrsg.) (2006): Betriebe ohne Betriebsrat. Informelle Interessenvertretung in Unternehmen, Frankfurt a. M./New York

Baethge, M. (1991): Arbeit, Vergesellschaftung, Identität. Zur zunehmenden normativen Subjektivierung von Arbeit, in: Soziale Welt 41 (1) S. 6-19

Baethge, M./Baethge-Kinsky, V. (1998): Jenseits von Beruf und Beruflichkeit? Neue Formen der Arbeitsorganisation und Beschäftigung und ihre Bedeutung für eine zentrale Kategorie gesellschaftlicher Integration, in: Mitteilungen aus der Arbeitsmarkt- und Berufsforschung 31 (3), S. $461-472$

Beck, U. (1986): Risikogesellschaft. Auf dem Weg in eine andere Moderne, Frankfurt a. M.

Becker, M. (unter Mitarbeit von Inéz Labucay und Cindy Kownatka) (2008): Optimistisch Altern. Theoretische Grundlagen und empirische Befunde demographiefester Personalarbeit für altersgemischte Belegschaften, München/Mering

Binswanger, M. (2010): Sinnlose Wettbewerbe. Warum wir immer mehr Unsinn produzieren, Freiburg

Blank, F. (2011): Soziale Rechte 1998-2005. Die Wohlfahrtsstaatsreformen der rot-grünen Bundesregierung, Wiesbaden

Boes, A./Kämpf, T. (2011): Global verteilte Kopfarbeit. Offshoring und der Wandel der Arbeitsbeziehungen, Berlin

Brinkmann, U./Dörre, K./Röbenack, S./Kraemer, K./Speidel, F.

(2006): Prekäre Arbeit. Ursachen, Ausmaß, soziale Folgen und subjektive Verarbeitungsformen unsicherer Beschäftigungsverhältnisse, hrsg. von der Friedrich-Ebert-Stiftung, Bonn

Bundesministerium für Familie, Senioren, Frauen und Jugend (BMFSFJ) (2011): 4. Bilanz Chancengleichheit. Erfolgreiche Initiativen unterstützen - Potenziale aufzeigen. Vierte Bilanz der Vereinbarung zwischen der Bundesregierung und den Spitzenverbänden der deutschen Wirtschaft zur Förderung der Chancengleichheit von Frauen und Männern in der Privatwirtschaft, Berlin

Bundesverband Betriebskrankenkassen (BKK) (2010): Arbeit und

Schlaf, BBK Faktenspiegel (11), Essen

Brehmer, W./Seifert, H. (2008): Sind atypische Beschäftigungsverhältnisse prekär? Eine empirische Analyse sozialer Risiken, in: Zeitschrift für ArbeitsmarktForschung 41 (4), S. 501-531

Brütt, Ch. (2011): Workfare als Mindestsicherung. Von der Sozialhilfe zu Hartz IV. Deutsche Sozialpolitik 1962 bis 2005, Bielefeld

Bundesagentur für Arbeit (BA) (2010): Beschäftigtenstatistik: Sozialversicherungspflichtige Bruttoarbeitsentgelte. Bericht der Statistik der BA - November 2010, Nürnberg

Bundesamt für Migration und Flüchtlinge (2011): Migrationsbericht 2009 des Bundesamtes für Migration und Flüchtlinge im Auftrag der Bundesregierung, hrsg. vom Bundesministerium des Innern, Berlin

Butterwege, Ch. (2006): Krise und Zukunft des Sozialstaates, Wiesbaden European Center for the Development of Vocational Training (CEDEFOP) (2008): Future skill needs in Europe. Medium-term forecast, Synthesis report, Luxemburg

Deutsche Angestellten-Krankenkasse DAK (2011): Gesundheitsreport 2011. Analyse der Arbeitsunfähigkeitsdaten. Schwerpunktthema: Wie gesund sind junge Arbeitnehmer?, Hamburg

Destatis (2009a): Bevölkerung Deutschlands bis 2060. 12. Koordinierte Bevölkerungsvorausberechnung. Begleitmaterial zur Pressekonferenz am 18. November in Berlin, hrsg. vom Statistischen Bundesamt, Wiesbaden
Destatis (2009b): Niedrigeinkommen und Erwerbstätigkeit. Begleitmaterial zum Pressegespräch am 19. August 2009 in Frankfurt a. M., hrsg. vom Statistischen Bundesamt, Wiesbaden

Dietz, M./Müller, G./Trappmann, M. (2009): Bedarfsgemeinschaften im SGB II: Warum Aufstocker trotz Arbeit bedürftig bleiben, IAB-Kurzbericht (2), Nürnberg

Dörre, K. (2003) Das flexibel-marktzentrierte Produktionsmodell Gravitationszentrum eines neuen Kapitalismus? in: Dörre, K./Röttger, B.(Hrsg.): Das neue Marktregime. Konturen eines nachfordistischen Produktionsmodells, Hamburg, S. 7-34

Edwards, R. C. (1979): Contested Terrain: The Transformation of the Workplace in the Twentieth Century, New York

Ellguth, P./Kohaut, S. (2011): Tarifbindung und betriebliche Interessenvertretung: Aktuelle Ergebnisse aus dem IAB-Betriebspanel 2010, in: WSI-Mitteilungen 64 (5), S. 242-247

Gottschall, K./Voß, G. G. (Hrsg.) (2003): Entgrenzung von Arbeit und Leben. Zum Wandel der Beziehungen von Erwerbstätigkeit und Privatsphäre im Alltag, München/ Mehring

Groß, H./Seifert, H. (Hrsg.) (2010): Zeitkonflikte. Renaissance der Arbeitszeitpolitik, Berlin

Hauser-Ditz, A./Hertwig, M./Pries, L. (2008): Betriebliche Interessenregulierung in Deutschland. Arbeitnehmervertretung zwischen demokratischer Teilhabe und ökonomischer Effizienz, Frankfurt a. M./New York Jürgens, K. (2011): Prekäres Leben, in: WSI-Mitteilungen 64 (8),

S. $379-385$

Jurczyk, K./Schier, M./Szymenderski, P./Lange, A./Voß, G. G. (2009): Entgrenzte Arbeit - entgrenzte Familie. Grenzmanagement im Alltag als neue Herausforderung, Berlin

Kalina, Th./Weinkopf, C. (2010): Niedriglohnbeschäftigung 2008: Stagnation auf hohem Niveau - Lohnspektrum franst nach unten aus, IAQ-Report 2010-06

Kleemann, Frank/Matuschek, I./Voß, G. G. (1999): Zur Subjektivierung von Arbeit. WZB-discussion paper p99-512, Berlin

Kratzer, N./Sauer, D. (2003): Entgrenzung von Arbeit. Konzepte, Thesen, Befunde, in: Gottschall, K./Voß, G. G. (Hrsg.) (2003): Entgrenzung von Arbeit und Leben. Zum Wandel der Beziehungen von Erwerbstätigkeit und Privatsphäre im Alltag. München/Mehring, S. 87-123 Kraemer, K. (2008): Prekarität - was ist das?, in: Arbeit 17 (2), S. 77-90

Möller, J. (2010): The German labor market response in the world recession - de-mystifying a miracle, in: Zeitschrift für ArbeitsmarktForschung 42 (4), S. 325-336

Promberger, M. (2006): Leiharbeit im Betrieb. Strukturen, Kontexte und Handhabung einer atypischen Beschäftigungsform. Abschlussbericht des Forschungsprojektes HBS-2002-418-3 (in Zusammenarbeit mit Bellmann, L./Dreher, Ch./Sowa, F./Schramm, S./Theuer, St.), Nürnberg Rau, R./Gebele, N./Morling, K./Rösler, U. (2010): Untersuchung arbeitsbedingter Ursachen für das Auftreten von depressiven Störungen. Abschlussbericht des Projektes F 1865, Bundesanstalt für Arbeitsschutz und Arbeitsmedizin, Dortmund/Berlin/Dresden

Sauer, D. (2010): Vermarktlichung und Vernetzung der Unternehmensund Betriebsorganisation, in: Böhle, F./Voß, G. G./Wachtler, G. (Hrsg.): Handbuch Arbeitssoziologie, Wiesbaden, S. 545-568

Schmidt, N. (2010): Auswirkungen des Strukturwandels der Wirtschaft auf den Bildungsstand der Bevölkerung, in: Wirtschaft und Statistik (6), S. $537-551$ 
Statistische Ämter des Bundes und der Länder (2009): Auswirkungen auf die Zahl der Erwerbspersonen, Statistisches Landesamt Baden-Württemberg, Demographischer Wandel in Deutschland (4), Stuttgart

Voß, G. G./Pongratz, H. J. (1998): Der Arbeitskraftunternehmer. Eine neue Grundform der Ware Arbeitskraft?, in: Kölner Zeitschrift für Soziologie und Sozialpsychologie (KZfSS) 50 (1), S. 131-158
Wetterer, A. (2003): Rhetorische Modernisierung: Das Verschwinden der Ungleichheit aus dem zeitgenössischen Differenzwissen, in: AxeliKnapp, G./Wetterer, A. (Hrsg.): Achsen der Differenz. Gesellschaftstheorie \& feministische Kritik II, Münster, S. 286-319

Wissenschaftliches Institut der AOK (WIdO) (2011): Burnout auf dem Vormarsch, Pressemitteilung des Wissenschaftlichen Instituts der AOK vom 19. April, Berlin 